\title{
Neuartiges Coronavirus (SARS-CoV-2)
}

Das sich von der zentralchinesischen Stadt Wuhan ausbreitende neue Coronavirus (SARS-CoV-2) stellt nach MERS (bislang 2494 gemeldete Infektionen in 27 Ländern mit einer Letalität von ca. $35 \%$ ) und SARS (8096 gemeldete Infektionen in 27 Ländern mit einer Letalität von ca. $10 \%$ ) die dritte durch Coronaviren (CoV) ausgelöste Epidemie im 21. Jahrhundert dar. Das volle Ausmaß sowohl der globalen Ausbreitung, als auch des durch SARS-CoV-2 ausgelösten klinischen Bildes (Coronavirus Disease 2019 (COVID-19)) ist bisher noch nicht vollständig abzusehen. Weitere Coronaviren sind bekannt, die vor allem in den Wintermonaten Auslöser von überwiegend milden Infektionen des oberen und gelegentlich des unteren Respirationstraktes sind. Endemisch in Deutschland und Europa sind dabei die humanen Coronaviren 229E, NL63, HKU1 und OC43. Die bisherigen Erkenntnisse zu SARS-CoV-2 sollen im Folgenden beschrieben werden (Stand 23.02.2020).

\section{Ausbreitung}

Seit dem 8. Dezember 2019 wurden in Wuhan, einer Elf-Millionen-EinwohnerMetropole und einem wichtigen Verkehrsknotenpunkt, zunehmend Patienten über ein Surveillance-System für Pneumonien unbekannter Ätiologie identifiziert, das infolge des SARS-Ausbruchs im Jahre 2003 etabliert wurde. Kriterien für Erkennung dieser Fälle waren neben dem fehlendem Erregernachweis: Fieber $\geq 38^{\circ} \mathrm{C}$, radiologischer Nachweis einer Pneumonie, niedrige oder normale Leukozytenzahl oder niedrige Lymphozytenzahl, ausbleibende Symptombesserung unter Antibiotikagabe für 3 bis 5 Tage. Die meisten Betroffenen arbeiteten auf oder lebten in der Nähe des „Huanan Seafood Market“, auf dem vor allem Fisch- und Fleischwaren, jedoch auch lebende Säugetiere und Vögel

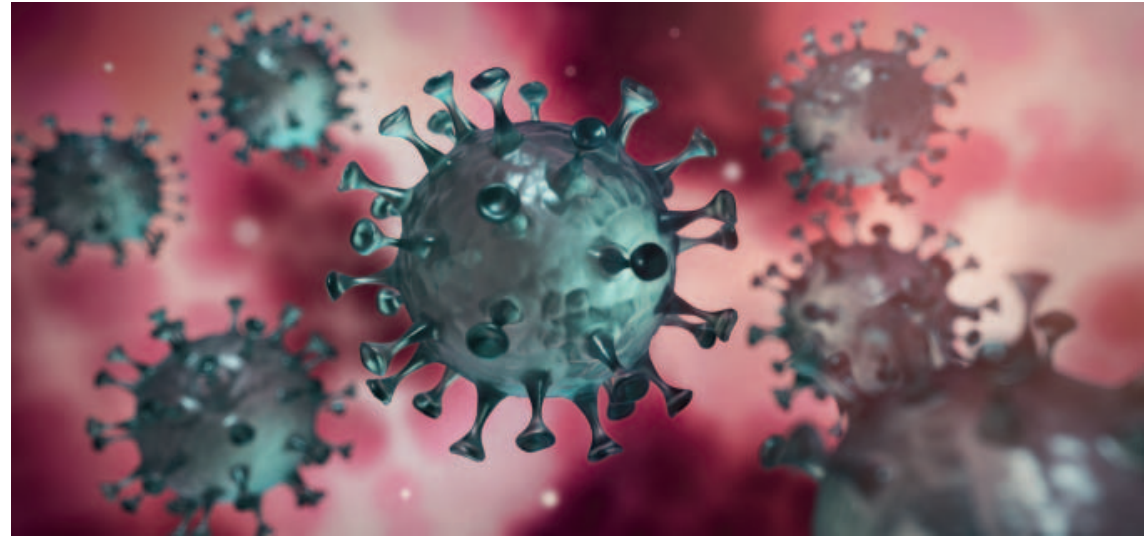

Das volle Ausmaß der globalen Ausbreitung von SARS-CoV-2 ist bisher noch nicht vollständig abzusehen. Symbolbild; Quelle: peterschreiber.media/Adobe Stock.

gehandelt wurden. Nachdem dieser epidemiologische Zusammenhang festgestellt wurde, erfolgte am 1. Januar 2020 die Schließung des Marktes durch die Behörden. Bis zum 3. Januar konnten 44 Patienten mit einer unklaren Pneumonie identifiziert werden. Als Ursache wurde durch moderne molekularbiologische Sequenzierverfahren SARS-CoV-2 bis zum 7. Januar identifiziert. Aufgrund der sehr schnell ansteigenden Zahl an Infektionen und der im Vergleich zu MERS- und SARS-CoV leichteren Mensch-zu-Mensch-Übertragung wurde Wuhan am 23. Januar unter Quarantäne gestellt. Zu diesem Zeitpunkt gab es weltweit 581 von der WHO bestätigte Fälle, 375 davon in der Provinz Hubei. Die Quarantäne wurde im Verlauf noch auf weitere Städte ausgedehnt. Der erste exportierte Fall außerhalb Chinas wurde am 13. Januar in Thailand berichtet. Die ersten Fälle in Deutschland, die gleichzeitig die ersten Mensch-zu-Mensch-Übertragungen außerhalb des chinesischen Festlandes darstellen, wurden Ende Januar in der Nähe von München diagnostiziert. Am 23.02.2020 betrug die Anzahl der von der WHO bestätigten Fälle 78811 in 29 Ländern, 1769 davon außerhalb von China und 2462 Todesfälle (davon 17 außerhalb Chinas).

\section{Reservoir}

Coronaviren können in verschiedenen Tierspezies nachgewiesen werden. Als natürliches Reservoir der neuartigen humanen Coronaviren wurden Fledermäuse identifiziert. Die Übertragung auf Menschen ist nach wie vor nicht im Detail geklärt. Es ist sehr wahrscheinlich, dass diese über Zwischenwirte vermittelt wird. Der Zwischenwirt für SARS-CoV-2 ist sehr wahrscheinlich das Schuppentier.

\section{Genomische Besonderheiten}

Das Genom SARS-CoV-2 zeigt die größte Homologie zu SARS-ähnlichen Viren der Fledermäuse. Im Vergleich zu den neuen, im Menschen gefundenen Coronaviren, zeigt es eine größere Homologie zu SARSCoV (ca. $79 \%$ ) als zu MERS-CoV (50\%). Besonders die Rezeptorbindungsregion ist eng mit der von SARS-CoV verwandt, was darauf hindeutet, dass von den Viren ebenfalls ACE2 als Zielstruktur auf den menschlichen Zellen genutzt wird.

\section{Klinische Aspekte}

Aufgrund der Identifikation der ersten Fälle über ein Surveillance-System für Pneumonien unbekannter Ätiologie wur- 
den zunächst schwere Verläufe beobachtet. Als initiale Symptome zeigten sich vor allem grippeartige Symptome wie Fieber, Husten, Kurzatmigkeit, Muskelschmerzen und Verwirrtheit. $90 \%$ der Patienten zeigten mehr als ein Symptom. Röntgenologisch zeigten sich vor allem bilaterale Pneumonien mit Milchglasphänomenen. Besonders schwere Infektionen mit der Entwicklung eines ARDS und der Notwendigkeit zur intensivmedizinischen Betreuung wurden vor allem bei Männern mit chronischen Vorerkrankungen oder einer Raucheranamnese beobachtet. Im Verlauf der Epidemie zeigten sich aber zunehmend auch sehr milde bzw. asymptomatische Verläufe und eine Angleichung des Anteils beider Geschlechter bei den Infizierten. Die Rate an schweren Infektionen betrug ca. $13 \%$ (definiert als Dyspnoe, Atemfrequenz über 30/min, Hypoxie, multiple pulmonale Infiltrate in der RöntgenThorax Aufnahme oder Zunahme des pulmonalen Infiltrats um $50 \%$ innerhalb von 24 bis 48 Stunden), die Letalität ca. 2,5\%.

\section{Falldefinition}

Die Falldefinition des Robert Koch Instituts umfasst zwei Personengruppen, bei denen eine spezifische Untersuchung auf SARSCoV-2 erfolgen muss: 1. Patienten mit respiratorischen Symptomen jeglicher Art und Kontakt zu einem bestätigtem Fall innerhalb der letzten 14 Tage. 2. Patienten mit einem akuten respiratorischen Syndrom, welches die unteren Atemwege betrifft, in Verbindung mit einem Aufenthalt in einem Risikogebiet in den letzten 14 Tagen. Als Hauptrisikogebiet gilt dabei die chinesische Provinz Hubei, in der auch Wuhan liegt.

\section{Diagnostik und Virusnachweis}

Serologische Tests zum Nachweis spezifischer Antikörper stehen noch nicht zur Verfügung. Der Nukleinsäurenachweis mittels PCR aus zwei oder drei verschiedenen Genregionen ist die Methode der Wahl. Derzeit ist über die genaue virale Ausscheidungskinetik an verschiedenen anatomischen Lokalisationen des Respira- tionstrakts noch nicht genug bekannt. Oropharyngeale Abstriche sowie Proben des unteren Respirationstrakts wie Trachealsekret oder bronchoalveoläre Lavageflüssigkeit (BAL) sind zu bevorzugen. Materialien des oberen Respirationstrakts können möglicherweise zu falsch negativen Ergebnissen führen. Bei begründetem weiter bestehendem Verdacht ist deshalb eine Verlaufsuntersuchung zu erwägen. Aufgrund der zeitlichen Überschneidung mit der „normalen“ Saison für virale respiratorische Atemwegserreger ist bei symptomatischen Patienten zusätzlich eine Testung auf Influenza- und Parainfluenzaviren und bei Kindern auch auf RSV und humanes Metapneumovirus anzustreben.

\section{Meldepflicht}

Der Verdacht und das Auftreten von respiratorischen Erkrankungen durch SARSCoV-2 ist nach $\S 7$ und 6 IfSG an das Gesundheitsamt meldepflichtig. Die Informationen werden dann nach Weitergabe an die Landesbehörden im Anschluss am Robert Koch Institut gebündelt.

\section{Therapie und Prophylaxe}

Aus Mangel an spezifischen Therapieoptionen steht bei schweren Infektionen in Analogie zu den Erfahrungen mit MERS-CoV und SARS-CoV die symptomatische Therapie in Verbindung mit intensivmedizinischen Maßnahmen bis hin zu speziellen Beatmungsverfahren beim Lungenversagen (ARDS) einschließlich extrakorporaler Membranoyxgenierung (ECMO) im Vordergrund der Behandlung. Es ist derzeit noch nicht abzusehen, ob, wann und in welcher Form ein spezifisches Virustatikum oder eine Impfung zur Verfügung stehen wird.

Bislang vorliegende experimentelle Daten legen nahe, dass die Arzneistoffe Remdesivir (ursprünglich gegen Ebola-Virus entwickelt) und Lopinavir/Ritonavir wirksam sein könnten. Aus dem SARS-Ausbruch 2002/03 ist bekannt, dass das HIV-Medikament Kaletra ${ }^{\circledR}$, das die Wirkstoffkombination Lopinavir/Ritonavir enthält, wirksam sein könnte. Eventuell lässt sich die Wirksamkeit durch die zusätzliche Gabe von Interferon-beta noch steigern. Remdesivir wurde in dem ersten USA-Fall therapeutisch eingesetzt [1]. Von der Gabe von Kortikosteroiden wird eher abgeraten.

An einem Impfstoff gegen SARS-CoV-2 wird fieberhaft gearbeitet. Auch im Idealfall dürften entsprechende Zulassungsstudien aber nicht vor Ende 2020 zu erwarten sein.

\section{WEITERFÜHRENDE LINKS}

Robert Koch-Institut. COVID-19 (Coronavirus SARS-CoV-2) (neuartiges Coronavirus). Im Internet: https:// www.rki.de/DE/Content/InfAZ/N/ Neuartiges_Coronavirus/nCoV.html European Centre for Disease Prevention and Control. Novel coronavirus. Im Internet: https://www.ecdc.euro pa.eu/en/novel-coronavirus-china World Health Organization. Novel Coronavirus (SARS-CoV-2) situation reports. Im Internet: https://www. who.int/emergencies/diseases/novelcoronavirus-2019/situation-reports/

Dr. Mario Hönemann, * Prof. Dr. Christoph Lübbert, DTM \& H, Prof. Dr. Uwe G. Liebert; Institut für Virologie und *Medizinische Klinik II, Bereich Infektions- und Tropenmedizin, Universitätsklinikum Leipzig, Zentrum für Infektionsmedizin

\section{Literatur}

[1] Holshue M, DeBolt C, Lindquist S et al. First Case of 2019 Novel Coronavirus in the United States. N Engl J Med 2020. doi:10.1056/ NEJMoa2001191

\section{Bibliografie}

DOI https://doi.org/10.1055/a-1113-3096 Online-Publikation: 24.2.2020 Dtsch Med Wochenschr 2020; 145: 336-337 (c) Georg Thieme Verlag KG, Stuttgart · New York ISSN 0012-0472 